\title{
Una propuesta de secuencia basada en el contexto, para la promoción de la argumentación científica en el aprendizaje de las reacciones químicas con estudiantes de educación media técnico profesional
}

\author{
Majorie Ibacache Plaza \\ Colegio Raúl Silva Henríquez: Ovalle.Chile.ip.marjorie@gmail.com
}

Cristian Merino Rubilar

Instituto de Quimica. Pontificia Universidad Católica de Valparaíso.Chile.cristian.merino@pucv.cl

[Recibido: 5 abril 2020. Revisado: 3 septiembre 2020. Aceptado: 20 septiembre 2020]

\begin{abstract}
Resumen: El propósito de este estudio fue analizar las limitaciones, posibilidades y condicionantes de una secuencia cuyo foco es la contextualización de los contenidos, abordando problemas reales y cercanos, permitiendo al estudiante poner en práctica la teoría en situaciones auténticas, promoviendo el pensamiento crítico y la argumentación científica, en estudiantes de Educación Media Técnico Profesional. Se trabajó con una muestra de 50 estudiantes (14-17 años) de un establecimiento educacional que imparte las carreras de Químico Industrial y Explotación Minera. La recolección de datos proviene de las actividades desarrolladas por los estudiantes respecto al el contenido de reacciones químicas bajo el contexto minero, para promover el desarrollo en la argumentación científica. Los resultados muestran que un alto porcentaje de los estudiantes, logran seleccionar una conclusión en base a pruebas y utilizar información de carácter disciplinar, socio-político, ético y ambiental al momento de elaborar una argumentación científica en un juego de roles.
\end{abstract}

Palabras clave: Argumentación científica; Enseñanza basada en el contexto; Reacción química; Educación media técnico profesional.

A context-based sequence proposal for the promotion of scientific argumentation in learning chemical reactions with high school technical professional students Abstract: This work analyzes the limitations, possibilities and conditioning factors of a sequence whose focus is
the contextualization of the contents, addressing real and close problems, allowing the student to put the theory
into practice in authentic situations, promoting critical thinking and argumentation scientific, in students of
Middle School Professional Technical. We worked with a sample of 50 students (14-17 years old) who study
Industrial Chemistry and Mining. Data collection comes from the activities in which students participated in the
content of chemical reactions under the mining context, to promote development in scientific argumentation.
The results show that a high percentage of the students manage to select a conclusion based on tests and use the
information of a disciplinary, socio-political, ethical, and environmental nature when preparing a scientific
argument in a role play.

Keywords: Scientific argumentation; Context-based teaching; Chemical reaction; Professional technical secondary education.

Para citar este artículo: Ibacache Plaza M., Merino Rubilar C. (2021) Una propuesta de secuencia basada en el contexto, para la promoción de la argumentación científica en el aprendizaje de las reacciones químicas con estudiantes de educación media técnico profesional. Revista Eureka sobre Enseñanza y Divulgación de las Ciencias 18(1), 1105. doi: 10.25267/Rev_Eureka_ensen_divulg_cienc.2021.v18.11.1105

\section{Introducción y contexto}

La Educación Media Técnico Profesional (EMTP), fue creada en Chile el año 1965 e implicó una reestructuración del sistema educativo, estableciéndose una educación general básica de ocho años y una educación media de 4 años para la modalidad científico humanista (EMCH) y la modalidad técnico profesional (EMTP), respectivamente (MINEDUC 2016). Actualmente la EMTP está orientada a la formación de 34 especialidades dirigidas a 15 sectores económicos 
las cuales les dan las alternativas al estudiantado de continuar su perfeccionamiento en institutos de formación técnica a nivel superior y/o universidades o de incorporarse a la vida laboral. En otros países latinoamericanos, esta formación se puede conocer bajo el nombre de ciclos formativos de grado medio (Santana, Feliciano y Santana-Lorenzo 2014).

La EMTP, tiene como finalidad preparar en forma íntegra a los estudiantes (14 a 18 años) para su posterior introducción inicial a la vida laboral, "Esta preparación se construye articulando el dominio de las competencias propias de una especialidad con el aprendizaje tanto de los objetivos transversales como de los objetivos y contenidos de la Formación General de la Educación Media" (MINEDUC, 2016, p.11). Hoy existen 945 liceos técnico-profesionales y la matrícula en EMTP representa el 39\% del total de estudiantes de todo el país. Sepúlveda y Valdebenito (2014) señalan que las expectativas de estos estudiantes, varían acorde a la educación recibida, por ejemplo el 50,6\% de los estudiantes se sienten preparados para enfrentarse a la Prueba de Selección Universitaria (PSU) versus el 83,4\% del estudiantado científico-humanista, y con ello acceder a estudios universitarios; en cuanto a los resultados nacionales del Sistema Nacional de Evaluación de Resultados de Aprendizaje (SIMCE 2015), los estudiantes que cursan la modalidad científico-humanista promedian 260,59 puntos en la prueba de lectura y 284,90 en la de matemáticas, mientras que aquellos que pertenecen a la modalidad técnico profesional promedian 230,59 y 233,65 puntos respectivamente. Se observa entonces, que los estudiantes de esta modalidad, se enfrentan a escenarios educativos donde el desempeño académico es en promedio inferior, con menos ambiciones educativas que sus contrapartes de la modalidad científico-humanista al igual que las expectativas que todos los actores poseen sobre su futuro logro educativo (Agencia de Calidad de la Educación 2016).

La información anterior, nos invita a preguntarnos, ¿cómo promover entonces ambientes de aprendizajes accesibles a los estudiantes que cursan la modalidad técnico profesional y dotar de nuevas experiencias a estos estudiantes? ¿A qué estrategias, propuestas y/o recursos puede recurrir un docente para que los estudiantes conecten con las especialidades que imparte su centro educativo? ¿Cómo el contexto donde se ven involucradas las especialidades de la modalidad EMTP impacta sobre la vida y las decisiones de los estudiantes frente a los sistemas de acceso a la universidad, vida laboral, la vida cotidiana, como por ejemplo en el área de la química?

Una plausible respuesta a las preguntas anteriores, podría ser, dar mayor énfasis a un enfoque basado en el contexto (Moraga, Espinet y Merino 2018), en este caso para la enseñanza de la química en la Educación Media Técnico Profesional y apostar por el desarrollo de la argumentación científica escolar, entendiendo que en la construcción del conocimiento científico, es de suma importancia la discusión y el contraste de ideas, además de la evolución del lenguaje retórico (Sardà y Sanmartí 2000). En esta misma línea, Izquierdo (2014) sugiere que en la enseñanza de la química, se debería optar por el desarrollo de una "actividad genuina", su argumento se sustenta en que sólo es posible evaluar los desempeños de los estudiantes "en acción" y a través de "genuinas preguntas". Así se lograría promover una reflexión que conecte la vida de los estudiantes y que les facilite la construcción de las respuestas a sus interrogantes; he aquí donde el contexto juega un rol fundamental, que puede nutrir y promover la observación, la reflexión, la discusión acerca de los fenómenos y con esto desarrollar la argumentación científica en los estudiantes. La argumentación científica escolar, permite que el estudiante a través de la escritura, el análisis de evidencias, orden de ideas y la integración de elementos incluyendo el tema en cuestión, contribuya al aprendizaje del contenido científico escolar (Revel Chion y Adúriz-Bravo 2014).

Dado las ideas anteriormente señaladas, se plantea una propuesta de secuencia de enseñanza aprendizaje basada en el contexto, donde el estudiante tenga la posibilidad de poner en 
práctica su conocimiento, y a su vez pueda desarrollar argumentos basados en pruebas o evidencias con respecto a las reacciones químicas que están involucradas en el contexto minero medioambiental y territorio tal como lo sugieren González et al. (2014).

A pesar de la diversidad de estudios en educación secundaria sobre aspectos de la enseñanza y aprendizaje de las reacciones químicas (Caamaño 2003, 2006, 2011), y sobre argumentación científica (Erduran y Jiménez-Aleixandre 2007), en la modalidad EMTP son escasos los estudios en relación a las limitaciones, posibilidades, condicionantes, y sustratos cualitativos sobre la enseñanza y aprendizaje de las ciencias. Si bien es posible encontrar estudios en el ámbito, estos apuntan hacia aspectos políticos, liderazgo y económicos (Larrañaga et al. 2013), entre otros; razón por la cual la pregunta que orienta este trabajo es: ¿qué posibilidades, limitaciones, condicionantes nos ofrece el contexto en la enseñanza de las reacciones químicas, y la promoción de la argumentación científica en los estudiantes que cursan la modalidad técnico profesional?

\section{Marco teórico}

Como marco teórico de este trabajo desarrollaremos aspectos sobre enseñanza basada en el contexto y argumentación científica escolar que son relevantes para esta investigación.

\section{Enseñanza basada en el contexto}

Según Gil y Vilches (2006), la incorporación de aspectos de relación ciencia-tecnologíasociedad-ambiente (CTSA) pueden favorecer y motivar al aprendizaje de las ciencias. Para los autores, la importancia de este foco viene dado porque la contextualización de los contenidos, trata de problemas reales y cercanos incentivando al debate y a la incorporación de la ciencia en la elaboración y resolución de problemas. Estas situaciones contextualizadas favorecen el pensamiento crítico de la ciudadanía (Hodson 1993). En esta misma línea, Onno de Jong (2006) para trabajar en el aula una enseñanza basada en el contexto (EBC), distingue cuatros dominios de contexto y su contribución en la formación de los estudiantes, a saber: a) desarrollo personal) p.e. cuidado de salud personal); b) social y de la sociedad (p. e., efectos de la lluvia ácida sobre el medio ambiente); c) práctica profesional (p. e., práctica de técnicos o ingenieros químicos), y d) científico-tecnológico (p. e., modelos y teorías históricas). Estos dominios concuerdan con lo que espera la EMTP del estudiante egresado. Caamaño (2011), enfatiza que desde el punto de vista teórico, la enseñanza contextualizada se fundamenta en el aprendizaje situado y asimismo Meroni, Copello y Paredes (2015), mencionan que esta aproximación permite que se promueva la comprensión de las ciencias como construcción humana, desarrollando y promoviendo la alfabetización científica.

\section{Argumentación científica}

Asociado a la contextualización, Zohar y Nemet (2002) indican que si se logran facilitar entornos donde se genere la argumentación científica, los estudiantes serían capaces de aprender con mayor facilidad los contenidos científicos, dado que lograrían correlacionarlos con su vida, acción que no necesariamente ocurre en la enseñanza tradicional. Como una forma de identificar características o factores clave relacionados con argumentación científica, en la tabla 1, se describen algunas investigaciones relevantes para este trabajo acerca del uso y promoción de la argumentación científica escolar. Se observa primordialmente que el modelo de preferencia para promover argumentos en el aula, corresponde al Modelo Argumentativo de Toulmin (MAT). La filosofía toulminiana (Toulmin 2003), establece que las ciencias están en constante transformación y se enfatiza en que la calidad de su enseñanza debe estar dirigida a las actitudes críticas con que los estudiantes aprenden a juzgar los conceptos, incluso los expuestos por sus docentes (Henao y Stipcich 2008). El MAT, está elaborado en base a los 
componentes básicos que debe poseer un buen argumento, los cuales son: a) la hipótesis; b) las evidencias; c) justificaciones; d) respaldos o fundamentos, y e) los refutadores que a su vez dan a lugar a los calificadores modales (Stincer y Blum 2017). Éstos últimos tienen el rol de matizar las conclusiones, para ello se utilizan palabras como: probablemente, necesariamente, presumiblemente, etc. (Mercado 2016).

Tabla 1. Investigaciones sobre los modelos que se utilizan para analizar argumentos (elaboración de los autores)

\begin{tabular}{|l|c|c|c|l|l|}
\hline \multicolumn{1}{|c|}{ Autor } & Año & n & Edades & \multicolumn{1}{|c|}{ Método } & \multicolumn{1}{c|}{ Algunos resultados } \\
\hline $\begin{array}{l}\text { Sanmartí, } \\
\text { Pipitone y } \\
\text { Sardá }\end{array}$ & 2009 & 30 & 16 & $\begin{array}{l}\text { Modelo } \\
\text { argumentativo de } \\
\text { Toulmin adaptado } \\
\text { por Sarda. }\end{array}$ & $\begin{array}{l}\text { Los estudiantes son capaces de desarrollar el } \\
\text { pensamiento crítico y evaluar la validez de la } \\
\text { información recogida desde internet. }\end{array}$ \\
\hline $\begin{array}{l}\text { Pérez y } \\
\text { Chamizo }\end{array}$ & 2013 & 45 & 20 y más & $\begin{array}{l}\text { Diagrama heurístico } \\
\text { en el desarrollo del } \\
\text { Modelo } \\
\text { argumentativo de } \\
\text { Toulmin }\end{array}$ & $\begin{array}{l}\text { Los estudiantes aprenden la importancia de } \\
\text { las explicaciones de los fenómenos que } \\
\text { observan en base a pruebas y no a partir de } \\
\text { creencias }\end{array}$ \\
\hline Mesa y Seña & 2013 & 40 & $9-11$ & $\begin{array}{l}\text { Modelo } \\
\text { argumentativo de } \\
\text { Toulmin }\end{array}$ & $\begin{array}{l}\text { Los estudiantes enriquecen continuamente } \\
\text { sus argumentos utilizando los elementos del } \\
\text { modelo argumentativo de Toulmin. }\end{array}$ \\
\hline $\begin{array}{l}\text { Stincer y } \\
\text { Blum }\end{array}$ & 2017 & 53 & 25 y más & $\begin{array}{l}\text { Modelo } \\
\text { argumentativo de } \\
\text { Toulmin }\end{array}$ & $\begin{array}{l}\text { Los estudiantes que elaboraron sus tesis bajo } \\
\text { el modelo de Toulmin se titularon a los tres } \\
\text { semestres de aprobados sus créditos } \\
\text { mientras que su contra parte lo hicieron } \\
\text { hasta después de tres años. }\end{array}$ \\
\hline
\end{tabular}

Adicionalmente, Sanmartí (2009 p. 256) señala que argumentar significa que un estudiante ha logrado desarrollar 5 aspectos relevantes, los cuales son:

1. Categorizar la pregunta planteada o la problemática.

2. Identificar los hechos o entidades que deben ser argumentadas.

3. Inferir posibles relaciones entre hechos o entidades argumentadas.

4. Seleccionar las relaciones más adecuadas.

5. Organizar las relaciones en forma coherente, diferenciando las ideas personales de las que tienen carácter científico. Redactar el texto relacionando causalmente los hechos y las ideas teóricas utilizando los conectores adecuados.

Por su parte Jiménez (2010), propone 10 ideas claves para desarrollar competencias en argumentación y el uso de pruebas y éstas son el sustento primordial en esta investigación las cuales contemplan:

1. Evaluar enunciados en base a pruebas.

2. La argumentación contribuye a competencias básicas y objetos generales de la educación

3. Las pruebas permiten distinguir conclusiones sustentadas en datos y no en opiniones.

4. Los argumentos comprenden conclusiones, pruebas y justificaciones.

5. Los criterios para evaluar pruebas incluyen especificidad, suficiencia, fiabilidad.

6. Los argumentos sobre modelos pretenden identificar relaciones causa-efecto.

7. La argumentación socio científica contribuye al pensamiento crítico.

8. Aprender a argumentar implica comunicar, persuadir a una audiencia.

9. El alumnado argumenta si su papel en clase lo requiere.

10. Un buen argumento tiene en cuenta los argumentos opuestos. 
Las ideas de estas autoras son clave para el diseño de una secuencia que recoja tanto los aspectos de contextualización como de argumentación para la enseñanza de la reacción química en la EMTP.

\section{Metodología}

El enfoque metodológico es de carácter mixto bajo un tipo de estudio correlacional cuasiexperimental, donde se estudiará la contextualización del contenido de reacciones químicas y la argumentación científica. Para el logro de este propósito, se analizó el progreso en los componentes de la argumentación según MAT y la calidad de los argumentos redactados por los estudiantes participantes del estudio.

\section{Participantes y contexto de la investigación}

Para comprender el contexto en el cual se desarrolla este trabajo (Oliva 2020), se debe saber que el establecimiento educacional donde se realizó la investigación lleva por nombre Colegio Raúl Silva Henríquez, está ubicado en la región minera de Coquimbo, en la comuna de Ovalle, funciona con una modalidad técnica profesional a nivel medio, donde se imparten las especialidades de Explotación Minera, perteneciente al Sector Minero y Química Industrial con mención en Laboratorio Químico, perteneciente al Sector Química e Industria, ambas relacionadas directamente con la industria minera. Actualmente consta de una matrícula de 350 estudiantes distribuidos desde primero a cuarto medio.

En la modalidad EMTP es fundamental relacionar los contenidos que se imparten tanto en el plan común, como en el plan diferenciado para que los estudiantes logren de forma óptima el perfil de egreso, además de ser capaces de emitir sus propios juicios basados en fundamentos teóricos y pruebas, habilidades que pueden ser reforzadas a través de la argumentación científica.

La muestra corresponde a 50 estudiantes de un total de 98 participantes iniciales. El criterio de selección responde a quienes participaron de todas las actividades aplicadas en la secuencia. Sus edades oscilan entre 14 y 17 años, su nivel socioeconómico es bajo. En la prueba SIMCE (2013) los resultados para la prueba de Lenguaje fue de 224 puntos y para matemáticas 218, mientras que como resultado de la prueba aplicada el año 2017 se obtuvo como puntaje 199 y 194 respectivamente. Estos resultados han llevado a clasificar al colegio como insuficiente según la Agencia de Calidad. Adicionalmente, el Índice de Vulnerabilidad (IVE) varía entre el $71,01 \%$ y el $100 \%$, por tanto, nos encontramos frente a un contexto educativo complejo donde no se evidencia un desarrollo de las habilidades de orden superior.

\section{Descripción de la secuencia}

El diseño de la secuencia contempló 5 actividades. Para ello, se consideraron: a) los estudios sobre las explicaciones de los estudiantes sobre reacciones químicas que podrían ocurrir en el contexto minero; b) identificar obstáculos en el aprendizaje de procesos químicos como la electro obtención de cobre, y finalmente; c) considerar aquellas ideas que están presentes en el contexto minero, vinculadas a las categorías medioambiental, social, disciplinar y ético. Para la enseñanza de las reacciones químicas, Kind (2004) sugiere que el diseño de actividades, se sustente en: a) establecer un punto de partida, b) usar modelos, y c) presentar ideas comunes de forma explícita. Lo anterior, es consistente con la contextualización de actividades relacionadas con la industria minera. Finalmente, se enfatizó en actividades para poner en práctica los conocimientos, a través del juego de roles.

El juego de rol o de simulación promueve situaciones que desde un punto de vista multidisciplinar, posibilita el análisis y la comprensión de los diferentes puntos de vistas e 
intereses en la toma de decisiones, ofreciendo estímulos para examinar modelos teóricos y aplicarlos en la vida cotidiana (Cook 2014, Smythe y Higgins 2007, Jackson y Walter 2000). Asimismo, argumentar es una estrategia para afrontar una situación problemática donde se necesita convencer a los demás que existen explicaciones más válidas y plausibles que otras (Sanmartí 2009). Es por ello que, es posible trabajar tanto la argumentación y el juego de roles como estrategias que contribuyen al aprendizaje activo de los estudiantes. Por tanto, se pensaron en actividades que demanden en el estudiante producir un texto con la finalidad de convencer a los demás frente a una problemática que involucra a las especialidades que conforman a su establecimiento educacional en relación al contenido de reacciones químicas.

Las actividades planificadas se presentan en la tabla 2. Se ha enfatizado en la recolección de pruebas para que los estudiantes puedan elaborar argumentos sólidos que cumplan con los componentes del modelo argumentativo de Toulmin (MAT). Los criterios para la organización y secuenciación de las actividades, subyacen en ciclo de aprendizaje (Sanmartí 2002), los cuales se señalan a continuación:

- La actividad para la explicitación y representación de las ideas iniciales, tiene como propósito observar imágenes de ecosistemas sin intervención y con modificaciones del hombre a través de yacimientos mineros para responder preguntas como por ejemplo: ¿qué diferencias observas en ambas imágenes? ¿crees que es posible que existan reacciones químicas en la industria minera?, si es así ¿en qué situaciones suceden? ¿cómo crees que una roca constituida por minerales de cobre puede transformarse en una olla de cobre?

- Para la introducción de nuevas variables, los estudiantes deberán localizar los yacimientos mineros en la región de Coquimbo e investigar los tipos de extracción de minerales y los procesos químicos involucrados.

- En la actividad de elaboración de conclusiones, se han propuesto tres actividades experimentales donde responderán preguntas diseñadas para la recolección de pruebas (Ver ejemplo de tipo de actividad en anexo 1), utilizando el formato las actividades para fomentar la argumentación, adaptado de Jiménez Alexandre (2009).

- Finalmente, para las actividades de transferencia a otros contextos, los estudiantes deberán formar grupos de trabajo donde responderán a una problemática desde la perspectiva de 4 roles los cuales corresponden a: 1) Pirquineros (minero que realiza las labores de extracción de mineral en forma artesanal y generalmente de manera independiente); 2) Grupo de medio ambientalistas (p. e., Greenpeace); 3) Representantes del sector agrícola; y 4) Habitantes del sector. Se simulará la apertura de un yacimiento minero en un sector cercano a la ribera del rio, a la ciudad y plantaciones agrícolas (véase ejemplo de tipo de actividad en anexo 2). 
Tabla 2. Matriz de planificación de actividades (elaboración de los autores).

\begin{tabular}{|c|c|c|c|c|}
\hline \# & Ciclo de aprendizaje & Contenido & AA & Actividad de evaluación \\
\hline 1 & Exploración & $\begin{array}{l}\text { Tipo de reacciones } \\
\text { químicas en la industria } \\
\text { minera }\end{array}$ & Clase & $\begin{array}{l}\text { Escala de Likert de MAT de } \\
\text { Toulmin, Niveles de } \\
\text { argumentación }\end{array}$ \\
\hline 2 & Introducción & $\begin{array}{l}\text { Industria minera en la } \\
\text { región y los minerales } \\
\text { que se extraen }\end{array}$ & Investigación & $\begin{array}{l}\text { Escala de Likert de MAT de } \\
\text { Toulmin, Niveles de } \\
\text { argumentación }\end{array}$ \\
\hline 3 & Estructuración & $\begin{array}{l}\text { Simulación de las } \\
\text { reacciones químicas que } \\
\text { ocurren en la industria } \\
\text { minera: } \\
\text { - Lixiviación. } \\
\text { - Extracción por } \\
\text { solventes. } \\
\text { - Electro-obtención }\end{array}$ & Laboratorio & $\begin{array}{l}\text { Escala de Likert de MAT de } \\
\text { Toulmin, Niveles de } \\
\text { argumentación, KPSI }\end{array}$ \\
\hline 4 & Aplicación & Industria minera & Juego de rol & $\begin{array}{l}\text { Escala de Likert de MAT de } \\
\text { Toulmin, Categorización de los } \\
\text { argumentos }\end{array}$ \\
\hline 5 & & Industria minera & Juego de rol & Rejilla \\
\hline
\end{tabular}

\section{Obtención de datos}

En este trabajo se distinguen 2 fuentes de datos, la primera corresponde a la organización y progresión de los componentes del MAT en las actividades 1, 2 y 3. La segunda fuente corresponde la detección de la calidad de los argumentos elaborados por los estudiantes mediante el juego de roles de las actividades 4 y 5 .

\section{Análisis de datos}

Para el análisis de datos, se revisaron los argumentos construidos por los estudiantes, localizando los componentes del MAT (adaptados por Mesa y Seña, 2013), considerando las actividades 1, 2 y 3 . Los elementos fueron del modelo fueron identificados como presente o ausente en función de: a) datos; b) respaldo a los datos; c) justificación; d) respaldo a la justificación; e) cualificador modal; f) refutación, y g) conclusión.

Para cuantificar la calidad de los argumentos escritos en las actividades 4 y 5 correspondiente al juego de roles, se utilizó como referencia una matriz propuesta por Henao y Stipcich (2008) que posee cuatro categorías: a) disciplinar; b) socio-político; c) ético, y e) ambiental. Los elementos se clasificaron en presente y coherente (véase la tabla 3). 
Tabla 3. Categorías propuestas para la identificación de los sustentos a los que recurren los estudiantes (Mesa y Seña 2013).

\begin{tabular}{|l|l|l|}
\hline \multicolumn{1}{|c|}{ Cualidad } & \multicolumn{1}{|c|}{ Presente } & \multicolumn{1}{c|}{ Coherente } \\
\hline Disciplinar & $\begin{array}{l}\text { La información se basa en } \\
\text { sustentos disciplinares propios de } \\
\text { las ciencias. }\end{array}$ & $\begin{array}{l}\text { Los sustentos disciplinares en los que los estudiantes } \\
\text { basan sus discursos son coherentes con el asunto } \\
\text { tratado. }\end{array}$ \\
\hline $\begin{array}{l}\text { Socio- } \\
\text { político }\end{array}$ & $\begin{array}{l}\text { La información se basa en } \\
\text { aspectos socio-políticos }\end{array}$ & $\begin{array}{l}\text { Los aspectos socio-políticos presentes en los } \\
\text { enunciados, son coherentes con el asunto tratado. }\end{array}$ \\
\hline Ético & $\begin{array}{l}\text { La información se basa en } \\
\text { aspectos éticos. }\end{array}$ & $\begin{array}{l}\text { Los aspectos éticos presentes en los enunciados son } \\
\text { coherentes con el asunto tratado. }\end{array}$ \\
\hline Ambiental & $\begin{array}{l}\text { La información se basa en los } \\
\text { aspectos ambientales }\end{array}$ & $\begin{array}{l}\text { Los aspectos ambientales presentes en los anunciados, } \\
\text { son coherentes con el asunto tratado. }\end{array}$ \\
\hline
\end{tabular}

Finalmente, para clasificar la calidad de los argumentos, se emplearon los siguientes cualificadores: notable, parcial o medio y baja o ausente (véase la tabla 4).

Tabla 4. Matriz de análisis de argumentos, adaptada de Henao (2010).

\begin{tabular}{|c|c|c|c|c|c|c|c|}
\hline \multicolumn{2}{|c|}{ Nivel de cualidad } & \multicolumn{2}{|l|}{ Notable } & \multicolumn{2}{|c|}{ Media / parcial } & \multicolumn{2}{|c|}{ Baja o ausente } \\
\hline $\begin{array}{l}\text { Pertinencia } \\
\text { Suficiencia }\end{array}$ & $\mathrm{P}$ & $\begin{array}{l}\text { La información } \\
\text { viene al caso, es } \\
\text { oportuna o } \\
\text { concerniente. } \\
\text { La información es } \\
\text { óptima. Atiende lo } \\
\text { requerido }\end{array}$ & $\begin{array}{l}\mathrm{P} \\
\mathrm{S}\end{array}$ & $\begin{array}{l}\text { Una parte de la } \\
\text { información viene } \\
\text { al caso o es } \\
\text { concerniente al } \\
\text { mismo. } \\
\text { No aplica término } \\
\text { medio }\end{array}$ & $\mathrm{Pp}$ & $\begin{array}{l}\text { La información } \\
\text { no se ajusta al } \\
\text { caso. } \\
\text { La información } \\
\text { no es óptima, } \\
\text { no atiende lo } \\
\text { requerido }\end{array}$ & $\mathrm{Np}$ \\
\hline $\begin{array}{l}\text { Necesidad } \\
\text { Coherencia }\end{array}$ & $\mathrm{N}$ & $\begin{array}{l}\text { La información es } \\
\text { relevante, } \\
\text { imprescindible } \\
\text { conveniente o muy } \\
\text { útil. } \\
\text { La información es } \\
\text { muy concordante o } \\
\text { tiene relación } \\
\text { explicita con la del } \\
\text { componente en } \\
\text { cuestión. }\end{array}$ & $\begin{array}{l}\mathrm{N} \\
\mathrm{C}\end{array}$ & $\begin{array}{l}\text { Una parte de la } \\
\text { información es } \\
\text { relevante, } \\
\text { imprescindible } \\
\text { conveniente o útil. } \\
\text { Sólo una parte de } \\
\text { la información es } \\
\text { concordante o hay } \\
\text { relación implícita } \\
\text { con la del } \\
\text { componente en } \\
\text { cuestión. }\end{array}$ & $\begin{array}{l}\mathrm{Pn} \\
\mathrm{Pc}\end{array}$ & $\begin{array}{l}\text { La información } \\
\text { es superflua o } \\
\text { irrelevante. } \\
\text { La información } \\
\text { no tiene } \\
\text { relación con la } \\
\text { del } \\
\text { componente en } \\
\text { cuestión. }\end{array}$ & $\begin{array}{l}\text { In } \\
\text { Ic }\end{array}$ \\
\hline Adecuada & $\mathrm{A}$ & $\begin{array}{l}\text { Desde el punto de } \\
\text { vista disciplinar, la } \\
\text { información es } \\
\text { apropiada, precisa, } \\
\text { completa y } \\
\text { actualizada. }\end{array}$ & $\mathrm{a}$ & $\begin{array}{l}\text { Desde el punto de } \\
\text { vista disciplinar, } \\
\text { sólo una parte de la } \\
\text { información es } \\
\text { apropiada. }\end{array}$ & $\mathrm{Pa}$ & $\begin{array}{l}\text { La información } \\
\text { no es apropiada } \\
\text { desde el punto } \\
\text { de vista } \\
\text { disciplinar. }\end{array}$ & $\mathrm{La}$ \\
\hline
\end{tabular}

\section{Resultados}

En la descripción de los resultados se analizan las respuestas de los estudiantes separado en: a) los componentes del MAT en las actividades 1, 2 y 3, b) para identificación de los sustentos y calidad de los argumentos, en las actividades 4 y 5 respectivamente. 


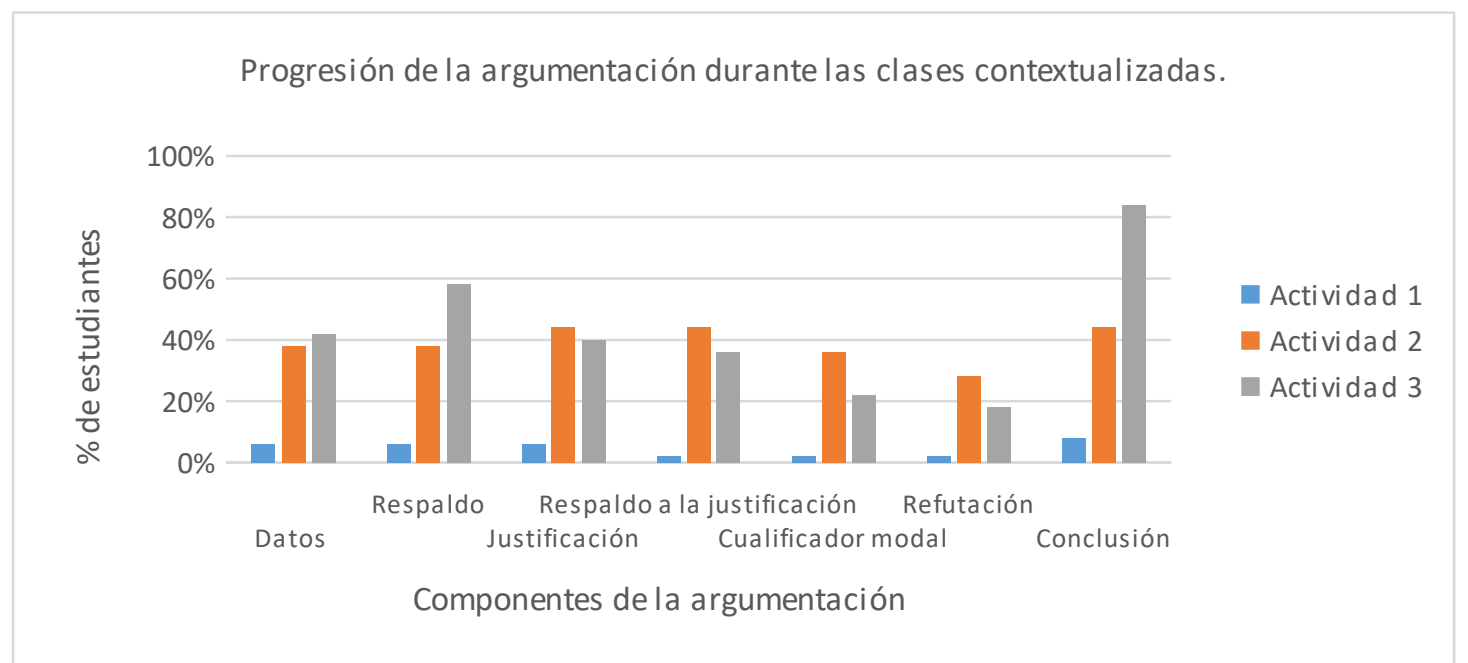

Figura 1. Resultados de la progresión de la argumentación según los componentes del MA, adaptado de Mesa y Seña (2013).

\section{Criterios que cumplen los estudiantes según MAT: actividades 1, 2 y 3}

Recordemos que para conocer la progresión en los componentes de la argumentación, nos basamos en el modelo argumentativo tulminiano, en términos de presencia o ausencia. Tras revisar los textos elaborados por los estudiantes, la figura 1 presenta los resultados obtenidos en las actividades 1,2 y 3 .

La figura 1, se observa un aumento significativo en el uso de datos por parte de los estudiantes, desde la primera actividad $(6 \%)$ para elaborar un argumento, avanzando hacia un $42 \%$ en la tercera actividad. En la primera actividad sólo el $6 \%$ fue capaz de redactar un respaldo sus datos, mientras que se observa un incremento hacia la tercera actividad el $58 \%$. En cuanto a la justificación al igual que en los componentes anteriores, el $6 \%$ es capaz de justificar, mientras que al transitar hacia la tercera actividad se observa un incremento del $40 \%$. En cuanto a la conclusión, en la primera actividad sólo el $8 \%$ fue capaz de seleccionar una conclusión en base a los datos, sin embargo en la actividad 3 el $84 \%$ fue capaz de hacerlo. Frente a esto podemos indicar que a medida que se realizan las tres actividades, se coincide con las 10 ideas claves sobre argumentación que propone Jiménez Aleixandre (2010). Los estudiantes progresan en forma ascendente frente al desarrollo de habilidades inferiores como es la observación o el uso de pruebas y logran elaborar argumentos sustentados por conclusiones, pruebas y justificaciones. A través de las actividades de laboratorio contextualizados, los estudiantes elaboran conclusiones construyendo su propio aprendizaje, poniendo en práctica algunos de los dominios señalados por De Jong (2006) como lo son los dominios de la práctica profesional y el dominio científico y tecnológico, en el cual, los estudiantes logran dimensionar en forma experimental como se obtiene el cobre a partir de los procesos químicos que sufre el mineral de cobre sulfurado.

\section{Calidad de los argumentos en el juego de roles: actividades 4 y 5}

Para la primera parte de la actividad 4, designada con el nombre ¿Qué hacemos con la minera en el rio Los Peñones?, los estudiantes se agruparon en cuatro roles simulando ser: a) un grupo de pirquineros, b) grupo medioambientalistas, c) habitantes del sector, y d) grupo del sector agrícola. Cada grupo trabajó el juego de roles, donde las instrucciones y reglas fueron las mismas. En la tabla 5 se presenta un ejemplo de las respuestas de los estudiantes en la actividad. 
Tabla 5. Componentes de la argumentación, juego de roles, pirquineros.

\begin{tabular}{|l|l|}
\hline Conclusión o propuesta & $\begin{array}{l}\text { Ayudar a los habitantes con una planta fotovoltaica que ayude a ahorrar un } \\
50 \% \text { de energía. }\end{array}$ \\
\hline Pruebas o datos & En Petorca se implementó un plan similar el cual fue acogido con éxito. \\
\hline Respaldo a datos & $\begin{array}{l}\text { Esta medida, ayudó a que la planta minera lograra ahorrar energía, cuidando } \\
\text { al medioambiente. }\end{array}$ \\
\hline $\begin{array}{l}\text { Justificación y respaldo a la } \\
\text { justificación }\end{array}$ & $\begin{array}{l}\text { La planta minera trabaja sin parar, lo que provoca un alto consumo de } \\
\text { energía eléctrica. }\end{array}$ \\
\hline Cualificador modal & Es por eso que \\
\hline Refutación & $\begin{array}{l}\text { La planta minera podría ser de gran ayuda en el ahorro de energía tanto para } \\
\text { los trabajadores como para los habitantes. }\end{array}$ \\
\hline
\end{tabular}

Para cuantificar la calidad de los argumentos escritos es que se utilizaron como referencia una rejilla propuesta por Henao y Stipcich (2008). En la tabla 6 se presenta un ejemplo de los argumentos clasificados como notables, elaborados por los estudiantes según sus categorías.

Tabla 6. Justificación y respaldo a la justificación en la calidad de los argumentos en la categoría disciplinar.

\begin{tabular}{|c|c|}
\hline Justificación & Respaldo a la justificación \\
\hline $\begin{array}{l}\text { "En la minería ocurren reacciones } \\
\text { químicas que pueden ser peligrosas } \\
\text { para el ser humano y el ecosistema". }\end{array}$ & $\begin{array}{l}\text { Hemos leído en las noticias que han existido derrames de } \\
\text { concentrado de cobre y de relave en el afluente del río Choapa, lo } \\
\text { cual podría provocar malformaciones fetales, cáncer, entre otros, esta } \\
\text { información la entrego el médico Andrei Tchernitchi de la } \\
\text { Universidad de Chile a la coordinadora por la defensa y derecho de } \\
\text { agua. }\end{array}$ \\
\hline
\end{tabular}

Los resultados de la calidad de los argumentos obtenidos en el juego de roles de acuerdo a las categorías ambiental, disciplinar, ético y sociopolítico, se sistematizan en la tabla 7.

Tabla 7. Calidad de los argumentos (elaboración por autores).

\begin{tabular}{|c|c|c|c|}
\hline $\mathrm{Am}$ & Disciplinar & Ético & Socic \\
\hline $\begin{array}{l}\text { Argumentos que tenga } \\
\text { que ver con el medio } \\
\text { ambiente, impacto } \\
\text { ambiental, agrupaciones } \\
\text { medioambientales, etc. }\end{array}$ & $\begin{array}{l}\text { Argumentos que estén } \\
\text { relacionado con el } \\
\text { concepto de reacciones } \\
\text { químicas en el ámbito } \\
\text { de la minería. }\end{array}$ & $\begin{array}{l}\text { Argumentos que } \\
\text { dirigen o valoran el } \\
\text { comportamiento } \\
\text { humano en una } \\
\text { comunidad }\end{array}$ & $\begin{array}{l}\text { Argumentos que involu- } \\
\text { cran a lo social y a lo polí- } \\
\text { tico, movimientos ciudada- } \\
\text { nos, etc., en este caso aso- } \\
\text { ciado al contexto minero y } \\
\text { las reacciones químicas. }\end{array}$ \\
\hline $\begin{array}{l}\text { El } 83 \% \text { de los } \\
\text { estudiantes utiliza datos } \\
\text { y los respalda en forma } \\
\text { notable. El } 67 \% \text { de los } \\
\text { estudiantes justifica u } \\
\text { respalda los datos en } \\
\text { forma parcial, el } 75 \% \\
\text { los refuta en forma } \\
\text { notable. Es decir, el } \\
\text { alumnado selecciona } \\
\text { información que puede } \\
\text { ser relevante en la } \\
\text { construcción de sus } \\
\text { argumentos temas } \\
\text { relacionados con temas } \\
\text { medioambientales con } \\
\text { respecto a la minería. }\end{array}$ & 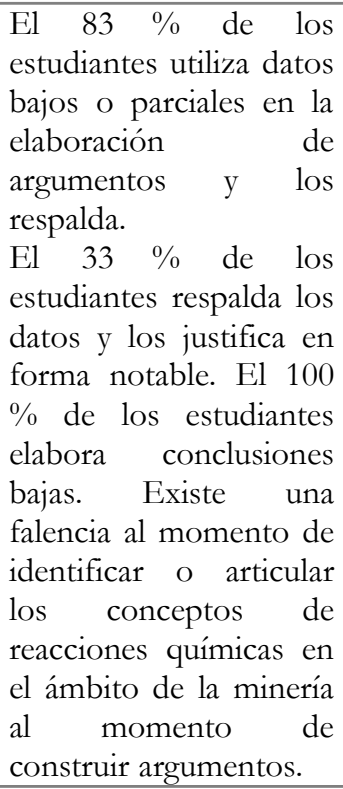 & $\begin{array}{l}\text { El } 83 \% \text { de los } \\
\text { estudiantes utiliza los } \\
\text { datos en forma } \\
\text { notable, mientras que } \\
\text { el } 100 \% \text { los respalda } \\
\text { en forma parcial. El } \\
84 \% \text { de los } \\
\text { estudiantes elabora } \\
\text { conclusiones en forma } \\
\text { parcial y notable. Los } \\
\text { estudiantes } \\
\text { seleccionan } \\
\text { información relevante } \\
\text { en cuanto a la } \\
\text { construcción r de } \\
\text { argumentos rar } \\
\text { involucra a la ética } \\
\text { entre las personas y el } \\
\text { contexto minero. }\end{array}$ & $\begin{array}{l}\text { El } 33 \% \text { de los estudiantes } \\
\text { utiliza los datos en la } \\
\text { elaboración de la } \\
\text { argumentación de forma } \\
\text { notable y asimismo el } 42 \% \\
\text { de ellos los respalda en } \\
\text { forma parcial. El } 83 \% \text { de } \\
\text { los estudiantes elabora } \\
\text { conclusiones, en forma } \\
\text { parcial y notable. Gran } \\
\text { parte de los estudiantes es } \\
\text { capaz de elaborar } \\
\text { argumentos en el plano } \\
\text { socio político en el } \\
\text { contexto minero, } \\
\text { considerando a los } \\
\text { diferentes actores } \\
\text { involucrados en el juego } \\
\text { de roles. }\end{array}$ \\
\hline
\end{tabular}

En la tabla 8 se presenta un ejemplo de cómo se cuantificó la vinculación en la calidad de los argumentos de los tres grupos que trabajaron bajo el rol de "medioambientalistas". 
Tabla 8. Ejemplificación de la vinculación de la calidad de los argumentos, adaptado de Mesa y Seña (2013).

\begin{tabular}{|c|c|c|c|c|c|c|c|}
\hline \multicolumn{2}{|l|}{ Saberes } & \multicolumn{6}{|c|}{ Características de los argumentos } \\
\hline & & Datos & $\begin{array}{c}\text { Respaldo a } \\
\text { datos }\end{array}$ & Justificación & Refutación & $\begin{array}{c}\text { Cualificador } \\
\text { modal }\end{array}$ & Conclusión \\
\hline \multirow[t]{3}{*}{ Disciplinar } & $\mathrm{B}$ & 33,3 & & 33,3 & & & $100 \%$ \\
\hline & $\mathrm{P}$ & 66,6 & & 33,3 & $100 \%$ & & \\
\hline & $\mathrm{N}$ & & $100 \%$ & 33,3 & & $100 \%$ & \\
\hline \multirow[t]{3}{*}{ Sociopolítico } & $\mathrm{B}$ & 66,6 & $100 \%$ & & & & \\
\hline & $\mathrm{P}$ & 33,3 & & $100 \%$ & $100 \%$ & & $100 \%$ \\
\hline & $\mathrm{N}$ & & & & & $100 \%$ & \\
\hline \multirow[t]{3}{*}{ Ético } & $\mathrm{B}$ & & & & $100 \%$ & & \\
\hline & $\mathrm{P}$ & & $100 \%$ & $100 \%$ & & & \\
\hline & $\mathrm{N}$ & $100 \%$ & & & & $100 \%$ & $100 \%$ \\
\hline \multirow[t]{3}{*}{ Ambiental } & $\mathrm{B}$ & & & & $100 \%$ & 100 & \\
\hline & $\mathrm{P}$ & & & $100 \%$ & & 100 & \\
\hline & $\mathrm{N}$ & $100 \%$ & $100 \%$ & & & $100 \%$ & $100 \%$ \\
\hline
\end{tabular}

\section{Discusiones y conclusión}

Por cuanto a las posibilidades en el uso de la contextualización y la argumentación en el diseño de actividades de enseñanza aprendizaje para EMTP, al recoger las argumentaciones de los estudiantes en sus actividades individuales (1, 2 y 3 ) relacionadas con las sesiones de laboratorio que incluyeron la lixiviación, la decantación y la electro obtención de cobre a partir de un mineral sulfurado, se logró cuantificar un aumento progresivo frente al cumplimiento de los componentes de la argumentación. Sin embargo, las condicionantes nos indican que es un proceso lento y que requiere de una aplicación constante y más aún cuando los estudiantes no han desarrollado habilidades básicas como lo es la observación, habilidad primordial en un práctico de laboratorio donde se pretende recoger datos para poder elaborar argumentos. La progresión de los componentes totales de los argumentos fue alcanzada por un intervalo entre un $18 \%$ y $58 \%$ de los estudiantes. No obstante, el aumento de los estudiantes que lograron seleccionar explicaciones o conclusiones en base a los datos o evidencias recogidas, aumento desde un $8 \%$ a un $84 \%$. Consideramos que es un avance significativo con respecto al desarrollo de habilidades que construyen conocimiento.

Otro condicionante es el anclaje del contenido disciplinar, vinculado con asuntos sociocientíficos. Para fomentar la argumentación científica se debe considerar que los temas a tratarse deben ser de interés de los estudiantes (Mesa y Seña 2013). En este caso, se seleccionó la minería en Chile ya que es un tema que aborda tanto a la especialidad de Química Industrial, como la de Explotación Minera, sabiendo que los estudiantes participes de la secuencia deberán seleccionar una de las dos en un par de años a futuro.

En cuanto a las posibilidades que nos ofrecen actividades grupales de juego de roles (4 y 5), el $83 \%$ de los estudiantes logra cumplir con los componentes de la argumentación, pero la calidad de los argumentos en las categorías disciplinar, socio-político, ético y ambiental fueron variando. Los argumentos con menor calidad fueron observados en la categoría disciplinar, donde gran parte de los estudiantes no profundizó el contenido de reacciones químicas con el objetivo planteado en el juego de roles. Los condicionantes que se observan, derivan de la categoría donde se obtuvieron una mayor cantidad de argumentos notables que fue en las dimensiones medio ambiente y en ética. Lo anterior, permite inferir que la argumentación científica y el juego de roles está directamente relacionada con la iniciación de los estudiantes en la alfabetización científica, factor clave para los estudiantes que cursan la EMTP. Ello, permitiría dimensionar de una manera clara cuáles son aquellos aspectos controversiales con 
los que deberán lidiar con sus futuros trabajos vinculados al sector minero y su entorno.

Frente a la búsqueda de información como prueba o evidencias para la argumentación en situaciones propias del entorno, se puede afirmar que los estudiantes recurren a diversas fuentes de información. Por ejemplo, lo que han escuchado u observado de su entorno, y las complementan con información obtenida desde la prensa, páginas oficiales o comparaciones similares ocurridas en otros sectores, acorde con lo indicado por Mesa y Seña (2013), "la enseñanza de la ciencia a partir de la discusión de asuntos sociocientíficos potencia la inclusión de conceptos en procesos en los que se privilegia la argumentación en el aula (p. 111)".

El hacer parte a los estudiantes de una enseñanza basada en el contexto, nos hemos aproximado a dominios que De Jong (2008) plantea. En específico: a) el dominio personal, donde se le han entregado herramientas a los estudiantes para que desarrollen opiniones personales frente a una problemática medioambiental; b) dominio social y de la sociedad, los estudiantes conocieron e investigaron información y recrearon situaciones frente a como la sociedad actúa frente a problemáticas medioambientales; c) dominio de la práctica profesional, los estudiantes replicaron a nivel micro escala los procesos químicos por los cuales es procesado el mineral sulfurado de cobre para la obtención de cobre puro, y finalmente; d) dominio científico y tecnológico, los estudiantes leyeron noticias locales e internacionales para robustecer su alfabetización científica sobre el tema de las reacciones químicas y la minería. Todo este progreso podría ser de vital importancia en las proyecciones que poseen los estudiantes que cursan la EMTP, ya que desarrollar habilidades propias de la argumentación, invitaría a los estudiantes apropiarse de ellas, repercutiendo en resultados de pruebas de evaluación nacional aumentando sus expectativas educativas, a diferencia de lo que es el escenario actual.

Para concluir y frente a la pregunta con la que iniciamos este artículo: ¿qué posibilidades, limitaciones y/o condicionantes los ofrece el contexto en la enseñanza de las reacciones químicas, y la promoción de la argumentación científica en los estudiantes que cursan EMTP? Podemos señalar que la contextualización nos ofrece atractivas posibilidades, frente a la progresión en el uso de los componentes de la argumentación. Además, se convierte en la puerta de entrada a la incorporación de la alfabetización científica al aula, invitando al estudiante TP desde el plan común hacia el plan diferenciado en alguna de las especialidades técnicas que seleccionará a futuro. También destacamos que la contextualización de los contenidos y dominios que los conforman (De Jong 2008) aportan en forma gradual en el desarrollo de los componentes de la argumentación científica. El condicionante a tener en cuenta es que para su adquisición sostenible, se debe estar diseñando actividades bajo este enfoque y aplicadas por un periodo prolongado de tiempo.

\section{Limitaciones}

Este estudio posee ciertas limitaciones relacionadas con la detección de habilidades de orden inferior necesarias para que el estudiantado construya el camino en la elaboración de argumentos, se aconseja fortalecer el desarrollo de la observación y evitar que los estudiantes describan los procedimientos en su lugar. A pesar de las limitaciones, la actividad contextualizada permitió que los estudiantes reforzaran la observación, investigación, lectura de noticias y la selección de fuentes fidedignas al momento de buscar información

\section{Agradecimientos}

Al proyecto Fondecyt 1180619 (ANID, Gobierno de Chile), al editor y revisores por sus aportaciones que han ayudado a robustecer este trabajo. 


\section{Referencias}

Agencia de la Calidad de la Educación (2016) Panorama de la educación media técnico profesional en Chile. Santiago: ACE.

Caamaño A. (2003) La enseñanza y el aprendizaje de la química. pp. 203-228 en M. P. Jiménez, Enseñar Ciencias. Barcelona: Graó.

Caamaño A. (2006) Retos del currículum de química en la educación secundaria. La selección y contextualización de los contenidos de química en los curriculum de Inglaterra, Portugal, Francia y España. Educación Quimica 17 (2),195-208.

Caamaño A. (2011) Enseñar química mediante la contextualización, la indagación y la modelización. Alambique. Didáctica de las Ciencias Experimentales 69, 21-34.

Cook D. (2014) Conflicts in Chemistry: The Case of Plastics, A Role-Playing Game for High School Chemistry Students.”Journal of Chemical Education 91(10), 1580-1586.

Jackson P., Walters J. (2000) Role-Playing in Analytical Chemistry: The Alumni Speak. Journal of Chemical Education 77(8), 1019-1025.

De Jong O. (2008) Context-based chemical education: how to improve it? Chemical Education International 8, 1-7.

Erduran S., Jiménez-Aleixandre M. (2007) Argumentation in Science education: Perspectives from classroom-based research. New York: Springer.

Jiménez M. (2010) 10 ideas claves. Competencias en argumentación y uso de pruebas. Barcelona: Graó.

Kind V. (2004). Más allá de las apariencias. Ideas previas de los estudiantes sobre conceptos básicos de química. México: Aula XXI/Santillana.

Gil V., Vilches A. (2006) Educación ciudadana y alfabetización científica: mitos y realidades. Revista Iberoamericana de Educación 42, 31-53.

González C., Merino C., Ahumada A., Arenas V., Bravo P. (2014) The local territory as a resource form learning science: A proposal for the design of teaching-learning sequence in science education. Procedia - Social and Behavioral Science 116, 4199-4204.

Henao B., Stipcich M. (2008) Educación en ciencias y argumentación: la perspectiva de Toulmin como posible respuesta a las demandas y desafíos contemporáneos para la enseñanza de las Ciencias Experimentales. Revista electrónica de Enseñanz̧a de las Ciencias 7, 47-62.

Hodson D. (1993) In search of a rationale for multicultural science education. Science Education 77 (6), 685-711.

Izquierdo M. (2014) Pasado y presente de la química: su función didáctica. pp. 13-36 en C. Merino, M. Arellano, A. Adúriz-Bravo, Avances en didáctica de la química: modelos y lenguaje. Valparaíso: Ediciones Universitarias Valparaíso.

Larragaña O., Cabezas G., Dussaillant F. (2013) Informe completo del Estudio de la Educación Técnico Profesional. Santiago: Programa de Naciones Unidas para el Desarrollo.

Martín-Páez T., Aguilera D., Perales F., Vílchez-González J. (2019) What are we talking about when we talk about STEM education? A review of literature. Science Education 103(4), 799-822.

Melo M., Hernández R. (2014) El juego y sus posibilidades en la enseñanza de las ciencias naturales. Innovación Educativa 14(66), 41-63. 
Mercado J. (2016) Un análisis del concepto de argumento en la teoría de la argumentación de Stephen Toulmin. (Tesis Doctoral). Cartagena: Universidad de Cartagena.

Meroni G., Copello M., Paredes J. (2015) Enseñar química en contexto. Una dimensión de la innovación didáctica en educación secundaria. Educación Química 26, 275-280.

Mesa S., Seña P. (2013) Argumentación en torno al concepto 'lo vivo': Discusiones sobre el maltrato animal como asunto sociocientifico. (Tesis Magister). Antoquía: Universidad de Antioquia.

Ministerio de Educación de Chile (2016) Bases Curriculares, Formación Diferenciada TécnicoProfesional, Especialidades y Perfiles de Egreso. Santiago: MINEDUC.

Moraga S., Espinet M., Merino C. (2018) El contexto en la enseñanza de la química: Análisis de secuencias de enseñanza y aprendizaje diseñadas por profesores de ciencias en formación inicial. Revista Eureka sobre Enseñanza y Divulgación de las Ciencias 16(1), 1604.

Oliva J. (2020) Sobre la importancia de contextualizar las investigaciones en didáctica de las ciencias. Revista Eureka sobre Enseñanza y Divulgación de las Ciencias 17(1), 1001.

Osborne J. (2009) Hacia una pedagogía más social en la educación científica: el papel de la argumentación. Educación química 20(2), 156-165.

Padilla C., Douglas S., López E. (2010) Competencias argumentativas en la alfabetización académica. Revista d' Innovació Educativa 4,1-12.

Pérez Y., Chamizo J. (2013) El ABP y el diagrama heurístico como herramientas para desarrollar la argumentación escolar en las asignaturas de ciencias. Ciência \& Educação (Bauru) 19(3), 499-516.

Pipitone C. (2009) Aprendiendo a argumentar entorno al concepto de cambio climático. pp. 1715-1719 en Enseñanza de las Ciencias, Numero Extra VIII Congreso Internacional sobre Investigación en Didáctica de las Ciencias. Barcelona: Universitat Autònoma de Barcelona.

Pizarro P., Santana A., Vial B. (2013) La participación de la familia y su vinculación en los procesos de aprendizaje de los niños y niñas en contextos escolares. Diversitas: Perspectivas en Psicología 9(2), 271-287.

Reyes F., Garritz A. (2006) Conocimiento pedagógico del concepto de "reacción química" en profesores universitarios mexicanos. Revista Mexicana de Investigación Educativa 11(31), 1175-1205.

Revel Chion A., Adúriz-Bravo, A. (2014) La argumentación científica escolar: Contribuciones a una alfabetización de calidad. Pensamiento Americano 7(13), 113-122.

Revel Chion A., Meinardi E., Adúriz-Bravo A. (2014) La argumentación científica escolar: contribución a la comprensión de un modelo complejo de salud y enfermedad. Ciência \& Educação (Bauru) 20(4), 987-1001.

Revel Chion A., Coulo A., Erduran S., Furman M., Iglesia P., Adúriz-Bravo, A. (2005) Estudios sobre la enseñanza de la argumentación científica escolar. Enseñanza de las Ciencias, Número Extra. VII Congreso Internacional sobre Investigación en Didáctica de las Ciencias 1-5. Barcelona: Universitat Autònoma de Barcelona.

Revetria J. (2015) Alfabetización Científica: Los Docentes de Ciencias y su Acción Didáctica. (Tesis Magister). Montevideo: Instituto Universitario.

Sardà A., Sanmartí N. (2000) Enseñar a argumentar científicamente: un reto de las clases de ciencias. Enseñanza de las Ciencias 18(3), 405-422.

Sanmartí N. (2009). Didáctica de las ciencias en la educación secundaria obligatoria. Madrid: Síntesis. 
Sanmartí N., Pipitone C., Sardá J. (2009) Argumentación en clases de ciencias. pp. 1709-1714 en Enseñanza de las Ciencias, Enseñanza de las Ciencias, Número Extra. VIII Congreso Internacional sobre Investigación en Didáctica de las Ciencias. Barcelona: Universitat Autònoma de Barcelona.

Santana L., Feliciano L., Santana-Lorenzo J. (2014). Madurez y autoeficacia vocacional en $3^{\circ}$ y $4^{\circ}$ de ESO, Bachillerato y ciclos formativos. REOP - Revista Española de Orientación y Psicopedagogía 24(3), 8-26.

Santibáñez C. (2014) ¿Para qué sirve argumentar?: Problematizando teórica y empíricamente el valor y la función de la argumentación. Círculo de Lingüistica Aplicada a la Comunicación 58, 163-205.

Sepúlveda L., Valdebenito, M.J. (2014) Aspiraciones y proyectos de futuro de estudiantes de enseñanza técnica-profesional: ¿Es pertinente un sistema diferenciado en la enseñanza media?. Polis (Santiago) 13(38), 597-620.

Smythe M., Higgins D. (2007) (Role) Playing Politics in an Environmental Chemistry Lecture Course. Journal of Chemical Education 84 (2), 241-244.

Stincer D., Blum B. (2017) El modelo argumentativo de Toulmin y la eficacia de titulación. Revista Electrónica de Investigación Educativa 19(4), 9-19.

Toulmin S. (2003) Los usos de la argumentación. Barcelona: Ediciones Península.

Trimiño-Quiala B., Voltarie-Basil R. (2013) La utilización de las ideas previas en el desarrollo de los conceptos: vías didácticas para su implementación en las clases de Ciencias Naturales. EduSol 13(44), 88-95.

Veneu F., Costa M. (2016) Temas controvertidos en la clase: ¿estamos listos? Una pequeña investigación entre los profesores de ciencias. Revista Electrónica Interuniversitaria de Formación del Profesorado 19(2), 89-101.

Zohar A., Nemet F. (2002) Fostering students' knowledge and argumentation skills through dilemmas in human genetics. Journal of Research in Science Teaching 39, 35-62. 
Anexo 1. Preguntas sobre la actividad experimental "Lixiviación” (elaboración de los autores).

Procedimiento experimental, lixiviación de un mineral de cobre sulfurado.

- Mide 5 gramos de mineral y adicionarlos en un vaso pp.

- Mide $50 \mathrm{~mL}$ de ácido sulfúrico 0,1 M y adiciónalos en el vaso pp que contiene el mineral y agite en forma constante por media hora.

- Retire el líquido formado y almacénelo hasta la próxima clase.

\section{Desarrollo.}

1. El líquido incoloro cambia a color calipso cuando interacciona con el mineral ¿Puedes explicar por qué sucede esto?

2. El mineral sulfurado de cobre está formado por varias sustancias, entre ellos se encuentra la calcosina $\left(\mathrm{Cu}_{2} \mathrm{~S}\right)$ y la covelina (CuS). ¿Por qué cambia de color el líquido?

3. Podemos creer en cualquiera de estas dos explicaciones:

\begin{tabular}{|l|l|}
\hline \multicolumn{1}{|c|}{ Explicación A } & \multicolumn{1}{c|}{ Explicación B } \\
\hline $\begin{array}{l}\text { Los componentes del mineral sulfurado son de color } \\
\text { calipso, éste se disuelve, cambiando el color del } \\
\text { líquido. }\end{array}$ & $\begin{array}{l}\text { Los átomos del mineral sulfurado interaccionan con el } \\
\text { ácido, reordenando los átomos. }\end{array}$ \\
\hline
\end{tabular}

Observa con detalle el procedimiento experimental y elige una de ambas explicaciones, anota las observaciones en las que te has basado para tu elección.

4. Justificación de las observaciones.

\begin{tabular}{|c|c|}
\hline Observación 1 & Observación 2 \\
\hline $\begin{array}{l}\text { "He observado que el mineral solido ha } \\
\text { interaccionado con el ácido sulfúrico, generando } \\
\text { burbujas y/o cambios de temperatura. Si_ No _ _ _Crees que esta observación es } \\
\text { correctamente explicada por } \mathrm{A} \text { ? } \\
\text { 1. } \mathrm{Si} \text { No__ Indica las razones. } \\
\text { 2. ¿Crees que esta observación es } \\
\text { correctamente explicada por } \mathrm{B} \text { ? } \\
\mathrm{Si} \text { No__ Indica las razones. }\end{array}$ & $\begin{array}{l}\text { "He observado que existe una formación de un nuevo } \\
\text { producto, diferente al inicial. } \\
\text { Si__ No__ } \\
\text { 1. ¿Crees_ que esta observación es } \\
\text { correctamente explicada por } \mathrm{A} \text { ? } \\
\mathrm{Si} \text { No__ndica las razones. } \\
\text { 2. ¿Crees que esta observación es } \\
\text { correctamente explicada por } \mathrm{B} \text { ? } \\
\mathrm{Si} \text { No_Indica las razones. }\end{array}$ \\
\hline
\end{tabular}

5. Información complementaria:

La ecuación química de la actividad experimental es:

$$
\mathrm{CuS}+\mathrm{Fe}_{2}\left(\mathrm{SO}_{4}\right)_{3} \rightarrow \mathrm{CuSO}_{4}+\mathrm{S}^{0}+2 \mathrm{FeSO}_{4}
$$

¿Crees que la explicación $\mathrm{A}$ es compatible con este dato? SI__ NO

Indica las razones.

¿Crees que la explicación $\mathrm{B}$ es compatible con este dato? $\mathrm{SI}$ Indica las razones

\section{Argumentación.}

A partir de la información recopilada escribe la conclusión seleccionada y arguméntala completando el siguiente recuadro

\begin{tabular}{|l|l|}
\hline Conclusión o propuesta. & \\
\hline $\begin{array}{l}\text { Pruebas (observación, hecho, experimento } \\
\text { que avala tu explicación seleccionada) }\end{array}$ & \\
\hline $\begin{array}{l}\text { Justificación (enunciado que relaciona tu } \\
\text { explicación con tus pruebas). }\end{array}$ & \\
\hline $\begin{array}{l}\text { Conocimiento básico (respaldo de la justificación } \\
\text { usando tu conocimiento teórico) }\end{array}$ & \\
\hline Cualificador modal & \\
\hline $\begin{array}{l}\text { Refutación (restricción o excepciones de tu } \\
\text { explicación seleccionada) }\end{array}$ & \\
\hline
\end{tabular}


Anexo 2. Actividad "juego de rol” (elaboración de los autores)

\section{Juego de rol}

\section{Para comenzar}

Lee con atención la siguiente nota:

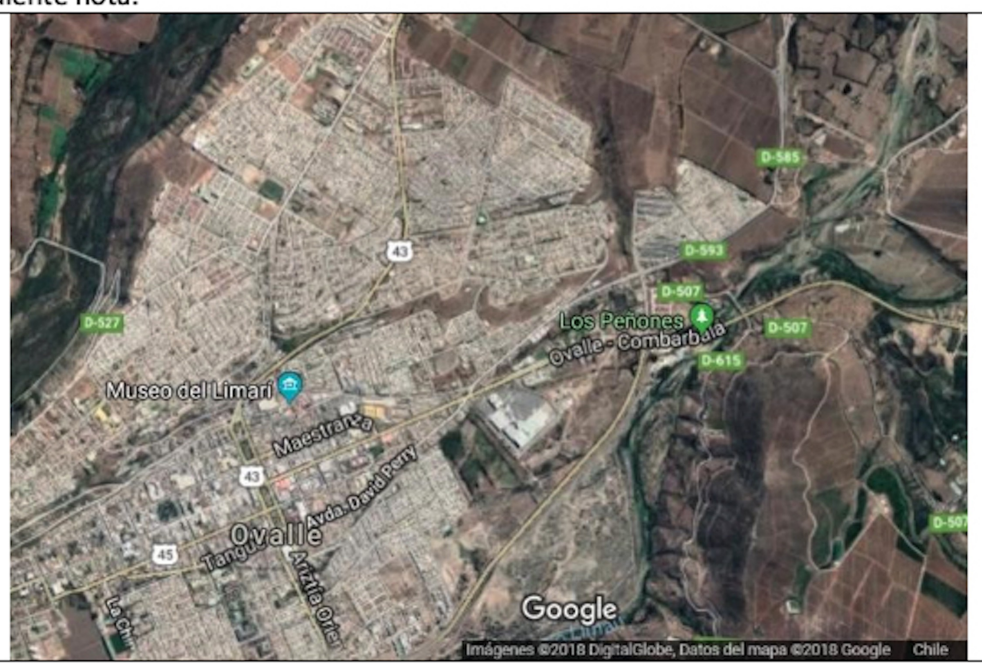

En los cerros aledaños al balneario los peñones se ha encontrado un yacimiento de mineral de cobre sulfurado, la cual ya fue concesionada. Esta noticia fue conocida por los pirquineros, las comunidades agrícolas (por ejemplo, granjeros), grupos medio ambientalistas y habitantes del sector, a las cuales se les pidió asistir a una mesa de trabajo donde los grupos deberán proponer ideas con la finalidad de convencer a los habitantes del sector para aprobar o no la explotación de la mina.

\section{Desarrollo}

Dirígete a la sala de computación e investiga la siguiente información, para ello tienes 45 minutos. Se sugiere establecer grupos de trabajo de 4 personas para optimizar el tiempo. A continuación:

- Identificación de roles de los integrantes del grupo (pirquinero, ambientalistas, granjeros, habitantes del sector)

- Busca antecedentes (noticias donde hayan participado grupos similares y las acciones que realizaron)

- Reflexiona sobre las ideas o pasos que han seguido los representantes del rol designado (puedes revisar conflictos anteriores, $\mathrm{y}$ las decisiones que se han tomado)

- Elabora una propuesta con respecto a qué hacer con frente al funcionamiento o no de la minera en el sector aledaño al balneario los peñones, no olvides considerar los tipos de reacciones químicas involucradas en el contexto minero, ético, medioambiental y sociopolítico.

- Para argumentar desde la postura de tu rol (pirquinero, ambientalistas, granjeros, habitantes del sector), a partir de la información recopilada escribe tu propuesta y arguméntala completando el siguiente recuadro.

\begin{tabular}{|l|l|}
\hline Rol & pirquinero, ambientalistas, granjeros, habitantes del sector..... \\
\hline Conclusión o propuesta. & \\
\hline $\begin{array}{l}\text { Pruebas (observación, hecho, } \\
\text { experimento que avala tu explicación } \\
\text { seleccionada) }\end{array}$ & \\
\hline $\begin{array}{l}\text { Respaldo a datos (enunciado que } \\
\text { relaciona tu explicación con tus pruebas). }\end{array}$ & \\
\hline $\begin{array}{l}\text { Conocimiento básico (justificación y } \\
\text { respaldo de la justificación usando tu } \\
\text { conocimiento teórico) }\end{array}$ & \\
\hline Cualificador modal & \\
\hline $\begin{array}{l}\text { Refutación (restricción o excepciones de } \\
\text { tu explicación seleccionada) }\end{array}$ & \\
\hline
\end{tabular}

